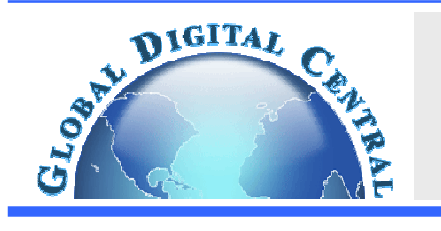

\title{
SORET AND DUFOUR EFFECTS ON MHD FLOW OF A MAXWELL FLUID OVER A STRETCHING SHEET WITH JOULE HEATING
}

\author{
B. Venkateswarlu ${ }^{a}$, P.V. Satya Narayana ${ }^{b^{*}}$ \\ a Department of Mathematics, Madanapalle Institute of Technoloty \& Science, Madanapalle, A.P., India \\ ${ }^{b}$ Department of Mathematics, SAS, VIT University, Vellore - 632014, T.N., India
}

\begin{abstract}
An analysis has been carried out to study the mixed convection flow, heat and mass transfer of an incompressible electrically conducting Maxwell fluid past a vertical stretching sheet in the presence of chemical reaction with thermal diffusion (Soret) and diffusion-thermo (Dufour) effects. The governing nonlinear partial differential equations along with the appropriate boundary conditions are non-dimensionalized using suitable similarity variables. The resulting transformed ordinary differential equations are then solved numerically by shooting technique with fourth order Runge Kutta method. The influence of various physical parameters on the flow, heat and mass transfer characteristics are discussed through graphs and tables. The results are physically interpreted. A comparison with the previously published results has been carried out and the results are found to be in good agreement. The conclusion is drawn that the flow field, rate of heat and mass transfer coefficients are significantly influenced by Dufour, Soret and Eckert numbers.
\end{abstract}

Keywords: Soret and Dufour numbers, Chemical reaction, MHD, Maxwell Fluid, Stretching Surface, Joule Heating

\section{INTRODUCTION}

The flow studies of non-Newtonian fluids attract a great deal of research in engineering and industry with its promising applications, such as the extrusion of polymers fluids, solidification of liquid crystals, animal bloods, exotic lubricants and colloidal suspension. Several fluids including polymer solutions, shampoo, paints etc. exhibit a non-linear relationship between the stress and rate of strain. There is a simplest subclass of the rate type fluids known as the Maxwell model. This model excludes the complicating effects of shear-dependent viscosity from any boundary layer analysis and enables, one to focus solely on the effects of fluids elasticity on the characteristics of its boundary layer (Sadeghy et al. 2005; Vieru et al. 2008; Hayat et al. 2008; Fetecau et al. 2009; Heyhat and Khabazi 2010). Examples of such fluids include glycerin, cruidoils or some polymeric solutions etc. Hayat et al. (2008) developed an analytic solution for unsteady MHD flow in a rotating Maxwell fluid through a porous medium. Tan and Masuoka (2007)) discussed linear convective stability of a Maxwell fluid layer in a porous medium. Some recent investigators (Abel et al. 2012; Mukhopadhyay 2012) dealt with the flows of Maxwell fluids.

The heat transfer analysis of MHD Maxwell fluid flow past a stretching sheet in the presence of thermal radiation effect can be quite significant. This is due to fact that many processes in engineering areas such as in design of reliable equipment, electrical power generation, nuclear plants, gas turbines and various propulsion devices or aircrafts, missiles, satellites. The influence of thermal radiation on MHD flow of a Maxwell fluid over a stretching sheet was discussed by Aliakbar et al. (2009). Hayat and Qasim (2010) investigated the influence of thermal radiation and Joule heating on MHD flow of a Maxwell fluid in the presence of thermophoresis. Hayat et al. (2011) have presented radiation effects on MHD flow of Maxwell fluid in a channel with porous medium. Thermal conductivity and thickness of the walls in the convection of viscoelastic Maxwell fluid layer was discussed by Perez-
Reyes and Davalos-Orozco (2011). Satya Narayana et al. (2015) have analyzed the effects of thermal radiation and heat source on MHD nanofluid past a vertical plate in a rotating system. MHD boundary layer flow of a Maxwell fluid past a stretching sheet in the presence of nanoparticles was investigated by Nadeem et al. (2014). Samir Kumar Nandy (2015) has studied an unsteady flow of Maxwell fluid in the presence of nanoparticles toward a permeable shrinking surface with Navier slip. Ramesh et al. (2016) discussed the stagnation point flow of Maxwell fluid towards a permeable surface in the presence of nanoparticles. Many researchers (Alizadeh-Pahlavan and Sadeghy 2009; Hayat et al. 2009 \& 2012; Abbas et al. 2010, Motsa et al. 2012; Abel et al. 2012) have studied the problem of Maxwell fluid flow over a stretching sheet analytically and numerically under different geometries.

The Maxwell fluid flow on MHD heat and mass transfer problems with chemical reaction is of great practical importance in many branches of science and engineering. The processes, such as drying, evaporation at the surface of a water body, energy transfer in a wet cooling the tower, and the flow in a desert cooler etc. Hayat et al. (2008) analyzed the MHD flow and mass transfer of UCM fluid past a porous shrinking sheet with chemical reaction species. Unsteady flow of a Maxwell fluid over a stretching surface in presence of chemical reaction is undertaken by Mukhopadhyay and Rama Subba Reddy (2012). Noor (2012) analyzed for MHD flow of a Maxwell fluid past a vertical stretching sheet in the presence of thermophoresis and chemical reaction. MHD flow of a Maxwell fluid past a vertical stretching sheet in the presence of thermophoresis and chemical reaction was presented by Stanford Shateyi (2013). Satya Narayana et al. (2013) investigated the Hall current and chemical reaction effects on MHD micropolar fluid in a rotating frame of reference. Chemical reaction and Radiation absorption effects on MHD micropolar fluid past a vertical porous plate in a rotating system presented by Satya Narayana et al. (2013). Several authors (Mukhopadhyay and Bhattacharyya 2012; Vajravelu et al. 
2012) have carried out their research work to investigate the effect of chemical reaction on some mass transfer related Maxwell fluid flow problems. Venkateswarlu and Satya Narayana (2015) analyzed the chemical reaction and radiation absorption effects on the flow and heat transfer of a nanofluid in a rotating system. Recently, Satya Narayana and Venkateswarlu (2016) have presented the heat and mass transfer on MHD nanofluid flow past a vertical porous plate in a rotating system.

Heat and mass transfer occur simultaneously between the fluxes and the driving potentials are of more intricate in nature. The energy flux caused by a composition gradient is called Dufour or diffusion-thermo effect and temperature gradients can create mass fluxes is called the Soret or thermal-diffusion effect. The thermal-diffusion and diffusionthermo effects are neglected on the basis that they are a smaller order of magnitude than the effects prescribed by Fourier's and Fick's laws. Bishnoi and Goyal (2012) have presented Soret Dufour driven thermosolutal instability of Darcy Maxwell fluid. Three dimensional flow of an UCM fluid under magnetic field was investigated by Hayat et al. (2012). Ramesh Chand and Rana (2014) have analyzed the double diffusive convection in a layer of Maxwell viscoelastic fluid in porous medium in the presence of Soret and Dufour effects. Bilal Ashraf et al. (2015) discussed three dimensional flow of Maxwell fluid with Soret and Dufour effects. Hayat et al. (2015) have examined Soret and Dufour effects in three dimensional flow of Maxwell fluid with chemical reaction and convective condition. Recently, Venkateswarlu and Satya Narayana (2016) investigated the influence of variable thermal conductivity on MHD Casson fluid flow over a stretching sheet with viscous dissipation, Soret and Dufour effects.

To the authors' knowledge (from the literature), no attempt has been made so far to analyze the Maxwell fluid flow, heat and mass transfer past a stretching surface with Dufour and Soret effects in the presence of chemical reaction and joule heating. The problem which is discussed here is a fundamental one that arises in many practical situations such as polymer extrusion process. Keeping this in view, the main objective of the present work is the numerical investigation of thermo diffusion and diffusion thermo effects over a stretching sheet with radiation and viscous dissipation. Similarity solutions are obtained and the reduced ordinary differential equations are solved numerically using shooting technique with Runge-Kutta integration scheme, which is more economical from the computational point of view. The effects of different flow parameters encountered in the equations are studied with the help of their graphical representations and tables.

\section{MATHEMATICAL FORMULATION OF THE PROBLEM}

We consider the two dimensional steady mixed convective heat and mass transfer of viscous incompressible boundary layer flow of a Maxwell fluid past a vertical sheet embedded in a porous medium. The origin of the system is located at the slit from which the sheet is drawn. The $x$-axis is considered in the direction along the sheet and the $y$-axis is normal to the surface. The flow is generated due to the stretching of sheet by applying two equal and opposite forces along the $x$-axis. A uniform transverse magnetic field of strength $B_{0}$ is imposed along the $y$ axis and the chemical reaction is taking place in the flow. We assume that magnetic Reynolds number of the fluid is small so that induced magnetic field and Hall effect may be neglected (which is a valid assumption on a laboratory scale). We also proposed that the plate is maintained at variable temperature and concentration, $T_{w}(x)>T_{\infty}$ and $C_{w}(x)>C_{\infty}$ respectively, where $T_{\infty}$ is the uniform temperature of the ambient fluid and $C_{\infty}$ is the uniform ambient concentration.

Under the usual boundary layer approximation, the governing equations describing the conservation of mass, momentum, energy and concentration in the presence of thermal radiation, Joule heating and chemical reaction are governed by the following equations.

$$
\begin{aligned}
& \frac{\partial u}{\partial x}+\frac{\partial v}{\partial y}=0 \\
& u \frac{\partial u}{\partial x}+v \frac{\partial u}{\partial y}+\lambda_{1}\left\{u^{2} \frac{\partial^{2} u}{\partial x^{2}}+v^{2} \frac{\partial^{2} u}{\partial y^{2}}+2 u v \frac{\partial^{2} u}{\partial x \partial y}\right\}=v \frac{\partial^{2} u}{\partial y^{2}} \\
& -\frac{v}{k} u-\frac{\sigma B_{0}^{2}}{\rho}\left(u+\lambda_{1} v \frac{\partial u}{\partial y}\right)+g\left\{\beta_{T}\left(T-T_{\infty}\right)+\beta_{C}\left(C-C_{\infty}\right)\right\} \\
& u \frac{\partial T}{\partial x}+v \frac{\partial T}{\partial y}=\frac{k}{\rho C_{P}} \frac{\partial^{2} T}{\partial y^{2}}-\frac{1}{\rho C_{P}} \frac{\partial q_{r}}{\partial y} \\
& +\frac{\mu}{\rho C_{P}}\left(\frac{\partial u}{\partial y}\right)+\frac{\sigma B_{0}^{2}}{\rho C_{P}} u^{2}+\frac{D_{m} k_{T}}{C_{S} C_{P}} \frac{\partial^{2} C}{\partial y^{2}} \\
& u \frac{\partial C}{\partial x}+v \frac{\partial C}{\partial y}=D \frac{\partial^{2} C}{\partial y^{2}}+\frac{D_{m} k_{T}}{T_{m}} \frac{\partial^{2} T}{\partial y^{2}}-k_{l}^{*}\left(C-C_{\infty}\right)
\end{aligned}
$$

The appropriate boundary conditions for the problems are given by

$$
\begin{aligned}
& \left.\begin{array}{l}
u=U_{w}(x)=a x \quad v=0 \quad T=T_{w}(x)=T_{\infty}+b x \\
C=C_{w}(x)=C_{\infty}+c x
\end{array}\right\} a t y=0 \\
& u \rightarrow 0 \quad \frac{\partial u}{\partial y} \rightarrow 0 \quad T \rightarrow T \quad C \rightarrow C_{\infty} \quad \text { as } y \rightarrow \infty
\end{aligned}
$$

The radiative heat flux $q_{r}$ is defined by the Rosseland approximation as follows

$$
q_{r}=-\frac{4 \sigma^{*}}{3 k^{*}} \frac{\partial T^{4}}{\partial y}
$$

The fluid phase temperature differences within the flow are sufficiently small so that $T^{4}$ may be described as a linear function of temperature. Hence, expanding $T^{4}$ in Taylors series about the free-stream temperature $T_{\infty}$ and neglecting higher order terms, we have

$$
\begin{aligned}
& T^{4} \cong 4 T_{\infty}^{3} T-3 T_{\infty}^{4} \\
& \frac{\partial q_{r}}{\partial y}=-\frac{16 \sigma^{*} T_{\infty}^{3}}{3 k^{*}} \frac{\partial^{2} T}{\partial y^{2}}
\end{aligned}
$$

Let us introduce the following non-dimensional quantities as:

$$
\begin{aligned}
& \eta=\sqrt{\frac{a}{v} y} u=a x f^{\prime}(\eta) \quad v=-\sqrt{a v} f(\eta) \quad \beta=a \lambda_{1} \quad R=\frac{4 \sigma^{*} T_{\infty}^{3}}{k k^{*}} \\
& S c=\frac{v}{D} \quad M=\frac{\sigma B_{0}^{2}}{a \rho} \quad K=\frac{v}{a k} \quad E c=\frac{u_{w}^{2}}{C_{P}\left(T_{w}-T_{\infty}\right)} \quad \theta(\eta)=\frac{T-T_{w}}{T_{w}-T_{\infty}} \\
& K r=\frac{k_{l}^{*}}{a} \operatorname{Pr}=\frac{\mu C_{P}}{k} \text { So }=\frac{b D_{m} k_{T}}{{ }^{v} T_{m}} \quad \operatorname{Re}_{x}=\frac{u_{w} x}{v} \quad \phi(\eta)=\frac{C-C_{w}}{C_{w}-C_{\infty}} \\
& D u=\frac{D_{m} k_{T}\left(C_{w}-C_{\infty}\right)}{v C_{P} C_{s}\left(T_{w}-T_{\infty}\right)} G r=\frac{g \beta_{T}\left(T_{w}-T_{\infty}\right) x^{3} / v^{2}}{u_{w}^{2} x^{2} / v^{2}} G m=\frac{g \beta_{C}\left(C_{w}-C_{\infty}\right) x^{3} / v^{2}}{u_{w}^{2} x^{2} / v^{2}}
\end{aligned}
$$


With the help of the above relations, the governing equations finally reduce to

$$
\begin{aligned}
& f^{\prime \prime \prime}+f f^{\prime \prime}-f^{2}+\beta\left(2 f f^{\prime \prime \prime}-2 f^{2} f^{\prime \prime \prime}\right)- \\
& \left(M+\frac{1}{K}\right) f^{\prime}+M \beta f f^{\prime \prime}+G r \theta+G m \phi=0 \\
& \frac{1}{\operatorname{Pr}}\left(1+\frac{4 R}{3}\right) \theta^{\prime \prime}+\left(f \theta^{\prime}-f^{\prime} \theta\right)+E c f^{\prime \prime 2}+M E c f^{2}+D u \phi^{\prime \prime}=0 \\
& \frac{1}{S c} \phi^{\prime \prime}+f \phi^{\prime}-f^{\prime} \phi+S 0 \theta^{\prime \prime}-K r \phi=0
\end{aligned}
$$

The boundary conditions (5) then become

$$
\begin{array}{lllll}
f=0 & f^{\prime}=1 & \theta=1 \quad \phi=1 & \text { at } \eta=0 \\
f^{\prime} \rightarrow 0 & f^{\prime \prime} \rightarrow 0 & \theta \rightarrow 0 & \phi \rightarrow 0 & \text { as } \eta \rightarrow \infty
\end{array}
$$

The physical quantities of engineering interest are skin friction coefficient, the local Nusselt number and the local Sherwood number.

Skin friction is caused by viscous drag in the boundary layer around the plate, and then, it is important to discuss the skin friction, from the knowledge of velocity, free skin friction form can be calculated as follows:

$$
C_{f}=\frac{\tau_{w}}{\rho U_{w}^{2} / 2} \Rightarrow C_{f} \operatorname{Re}_{x}^{1 / 2}=-2 f^{\prime \prime}(0)
$$

Where the skin friction $\tau_{w}=\mu\left\{\frac{\partial u}{\partial y}\right\}_{y=0}$

The rate of heat transfer between the fluid and the plate is studied through non-dimensional Nusselt number. The rate of heat transfer in terms of Nusselt number is given by

$$
N u_{x}=\frac{x q_{w}}{k\left(T_{w}-T_{\infty}\right)} \Rightarrow \frac{N u_{x}}{\operatorname{Re}_{x}^{1 / 2}}\left\{\frac{4}{4+3 R}\right\}=-\theta^{\prime}(0)
$$

Where the wall heat flux is $q_{w}=-\left\{\left(k+\frac{16 \sigma^{*} T_{\infty}^{3}}{3 k^{*}}\right) \frac{\partial T}{\partial y}\right\}_{y=0}$

The local Sherwood number $S h$ is given by

$$
S h=\frac{x j_{w}}{D\left(C_{w}-C_{\infty}\right)} \Rightarrow \frac{S h}{\operatorname{Re}_{x}^{1 / 2}}=-\phi^{\prime}(0)
$$

Where the wall mass flux from the plate is $j_{w}=-\left\{\frac{\partial C}{\partial y}\right\}_{y=0}$

It is worth mentioning that in the absence of magnetic parameter, porous parameter, Grashof number and modified Grashof number the equation (2) of present study reduce to equation (11) of Sadeghy et al. (2005), while in the absence of Maxwell parameter the equations reduce to those of Chen and Chav (1998) for different physical situations.

\section{NUMERICAL PROCEDURE}

The equations (10)-(12) are extremely coupled non-linear ordinary differential equations of third order and second order, respectively. Analytical solutions are not possible for the complete set of equations and hence we solved numerically by most efficient fourth-order Runge-
Kutta integration scheme with shooting method. The utmost vital factor of this method is to choose the appropriate finite values of $\eta \rightarrow \infty$. In order to determine $\eta_{\infty}$ for the boundary value problem stated by equations (10)-(12), we start with some initial guess value for some particular set of physical parameters to obtain $f^{\prime \prime}(0)$. The solution

procedure is repeated with another large value of $\eta_{\infty}$ until two successive values of $f^{\prime \prime}(0)$ differ only by the specified significant digit. The last vale of $\eta_{\infty}$ is finally chosen to be the most appropriate value of the limit $\eta \rightarrow \infty$ for the particular set of parameters. The value of $\eta$ may change for another set of physical parameters. Once the finite value of $\eta$ is determined then the coupled boundary value problem given by equations (10), (11) and (12) are solve numerically using the shooting method. In this method the third-order non-linear equation (10) and second-order equations (11), (12) have been reduced to seven simultaneously ordinary differential equations for seven unknowns. In this way all the six initial conditions are determined. Now it is possible to solve the resultant system of equations by employing shooting technique with Runge-Kutta scheme.

\section{RESULT AND DISCUSSION}

The numerical solutions are obtained for velocity, temperature, concentration, the skin friction coefficient, the local Nusselt number, and the local Sherwood number for different values of governing parameters. The obtained results are displayed through figures and Tables. The accuracy of the present work has been validated by comparing the values of skin friction co-efficient $f^{\prime \prime}(0)$ with the reported results of Subhas Abel et al. (2012) and Sadgehy et al. (2005). It is witnessed that our results are in good agreement with the results obtained by the previous researchers as seen from the tabulated values in Table 1.

Table 1: Comparison of the skin-friction co-efficient $f^{\prime \prime}(0)$ for different values of $\beta$ with Sadgehy et al.[34], Subhas Abel et al. (2011).

\begin{tabular}{|c|c|c|c|c|}
\hline$\beta$ & $\begin{array}{c}\text { Sadgehy } \\
\text { et al. } \\
(2005)\end{array}$ & $\begin{array}{c}\text { Subhas } \\
\text { Abel et al. } \\
(2009)(\mathrm{A})\end{array}$ & $\begin{array}{c}\text { Present } \\
\text { Study (B) }\end{array}$ & $\begin{array}{c}\text { Percentage of } \\
\text { error }\left|\frac{B-A}{B}\right|\end{array}$ \\
\hline 0 & -1.0000 & -0.9999 & -0.999963 & 0.0063 \\
0.4 & -1.1008 & -1.1018 & -1.101849 & 0.0049 \\
0.6 & -1.0015 & -1.1501 & -1.150164 & 0.0064 \\
\hline
\end{tabular}

Effects of Deborah number/Maxwell parameter $\beta$ on velocity, temperature and concentration profiles are shown in figures 1(a), 1(b), and 1(c) respectively. From figure 1(a), it is seen that an increase in $\beta$ leads to a decrease in the velocity and hence the boundary layer thickness decreases. This is due to the fact that the increase of $\beta$ leads to thinning of the intrinsic fluidity of the material. It is obvious from figures 1(b) and 1(c) that the temperature field increases and concentration distribution decreases by increasing Maxwell parameter $\beta$. These results are same as noted in the Ref. (Subhas Abel et al., 2009).

Figures 2(a)-2(c), respectively, show the velocity, temperature and concentration distributions for different values of Eckert number $E c$. We have considered viscous dissipation term to increase the fluid motion. From this term we obtained dimensionless parameter $E c$. This parameter is called the fluid motion controlling parameter. From figures 2(a) and 2(c), it is observed that, the velocity and concentration profiles decrease with the increase of Eckert number $E c$. Whereas reverse trend is observed in the case of temperature profile (fig. 2(b)). This is due to the fact that heat energy is stored in the liquid due to the frictional 
heating. Thus the effect of increasing $E c$ is to enhance the temperature at any point in the thermal boundary layer.

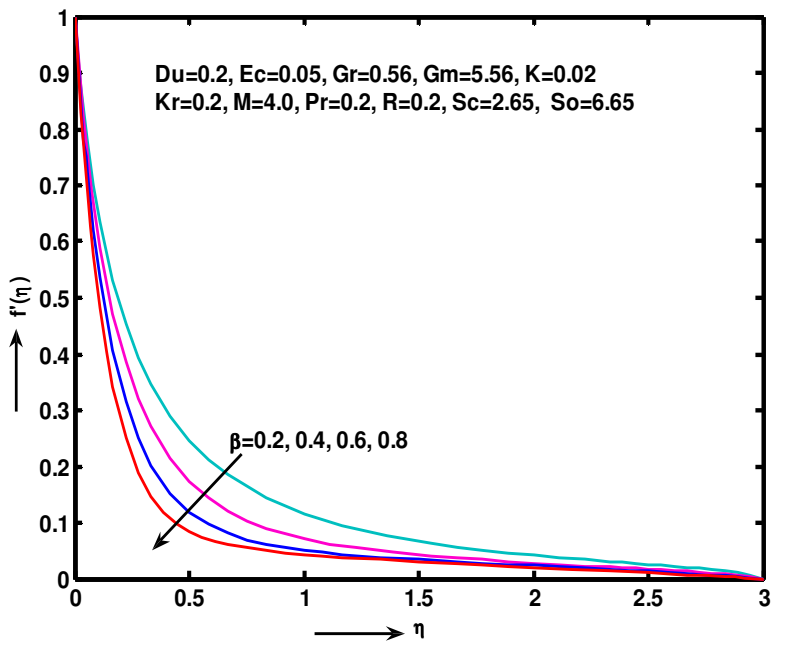

(a)

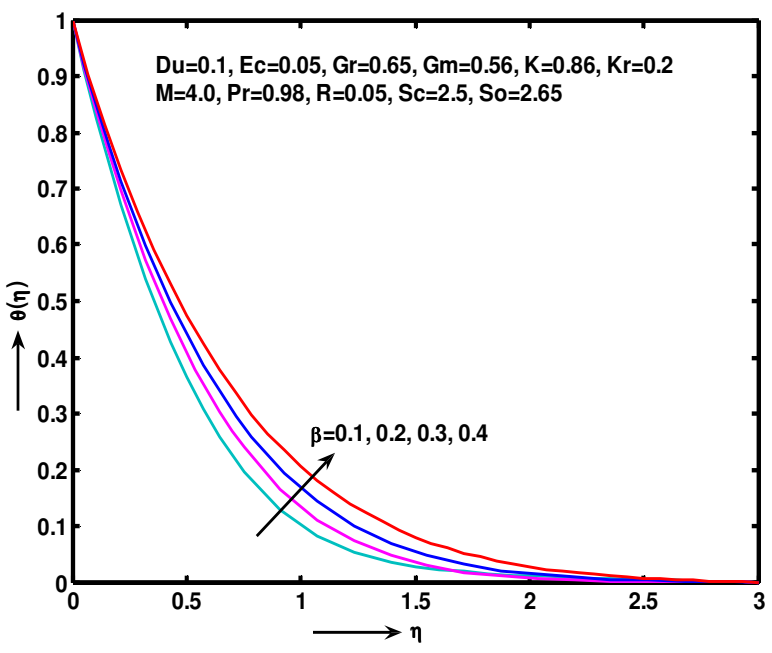

(b)

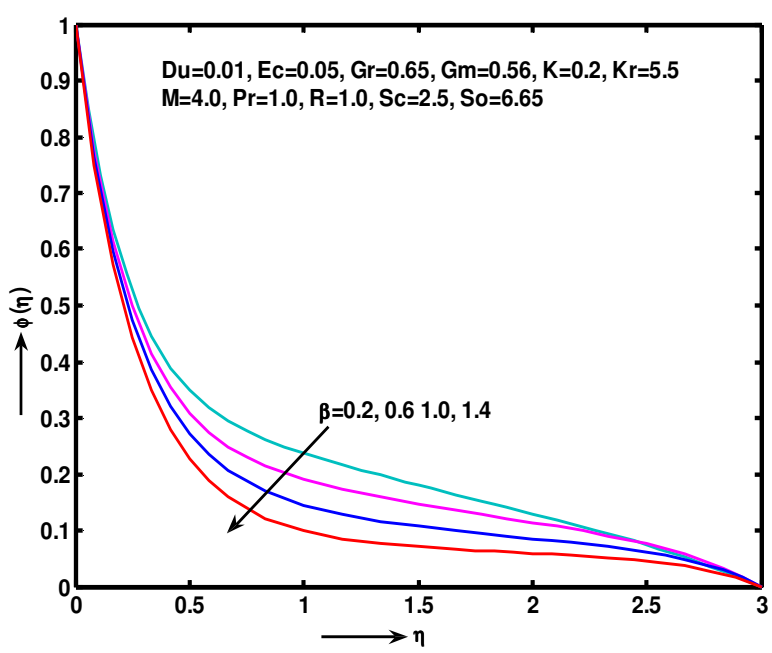

(c)

Fig. 1 Effect of $\beta$ on (a) Velocity (b) Temperature (c) Concentration profiles

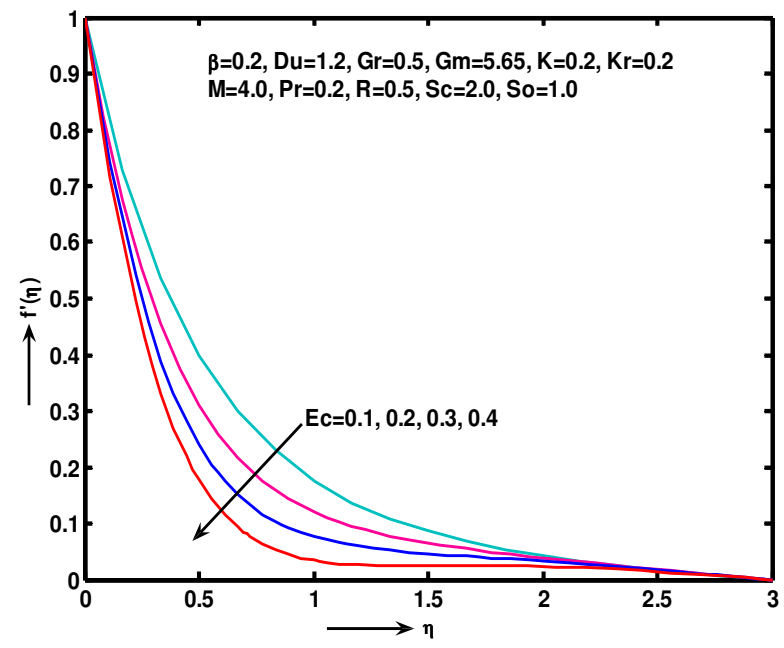

(a)

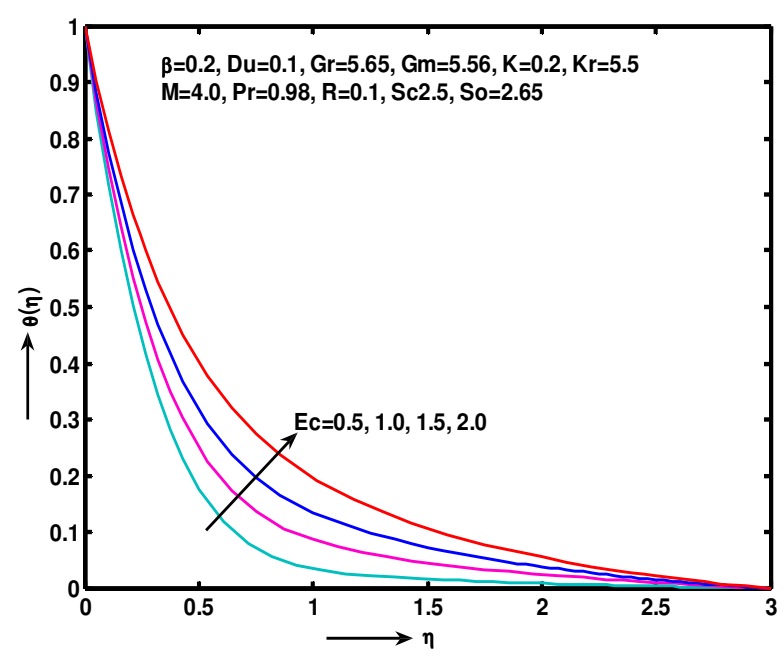

(b)

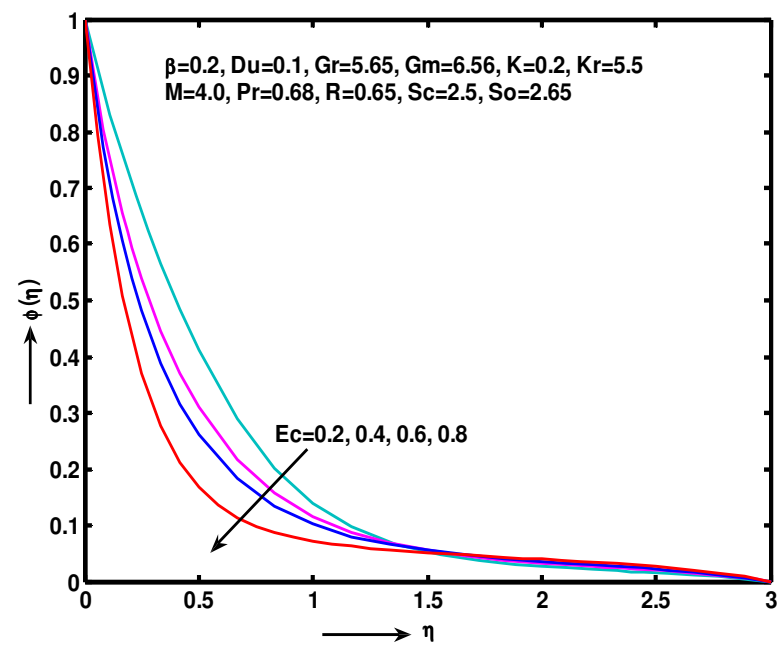

Fig. 2 Effects of $E c$ on (a) Velocity (b) Temperature (c) Concentration profiles

Figures 3(a), 3(b), and 3(c), respectively, show the velocity, temperature and concentration distributions for different values of the chemical reaction parameter $K r$. It is clear from the figures that the 
velocity and concentration profiles decrease with the increase of the parameter $K r$ whereas the reverse trend is observed in the case of temperature profile. This is due to the dominating role of the Soret and Dufour in the flow field. These results clearly supported from the physical point of view.

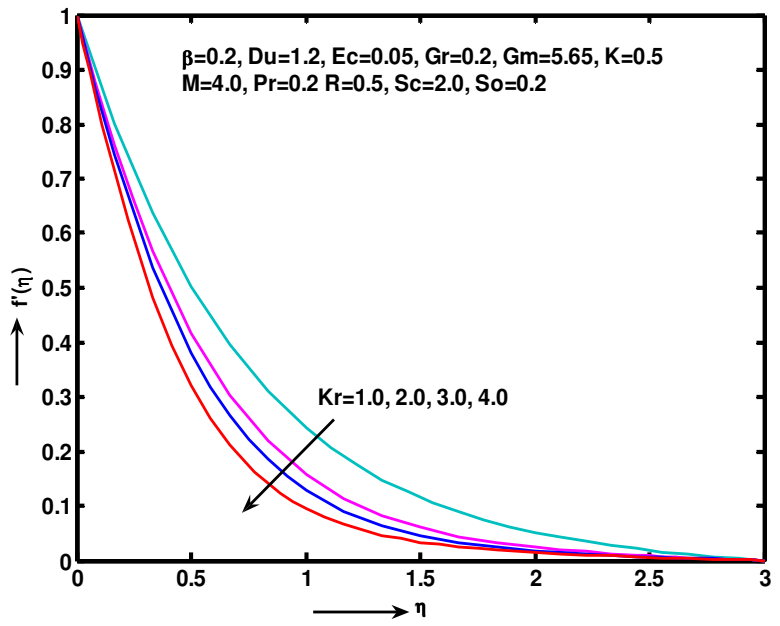

(a)

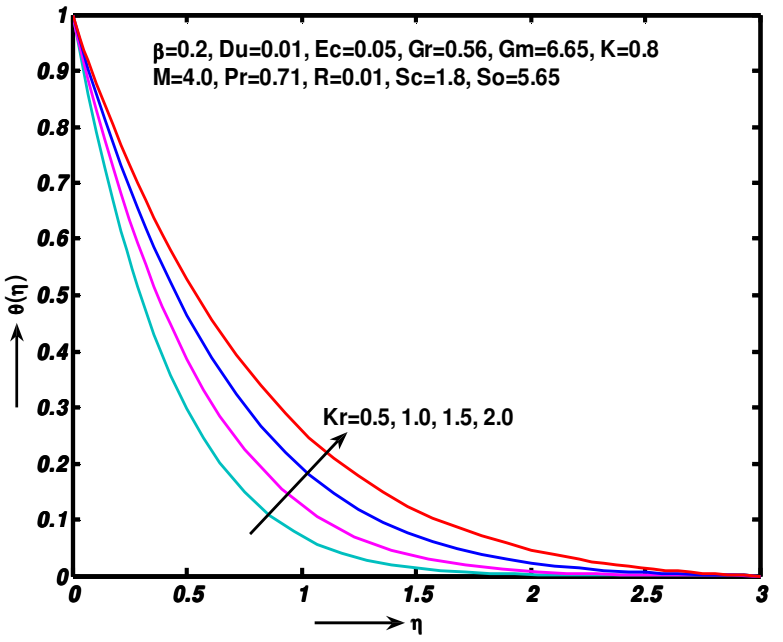

(b)

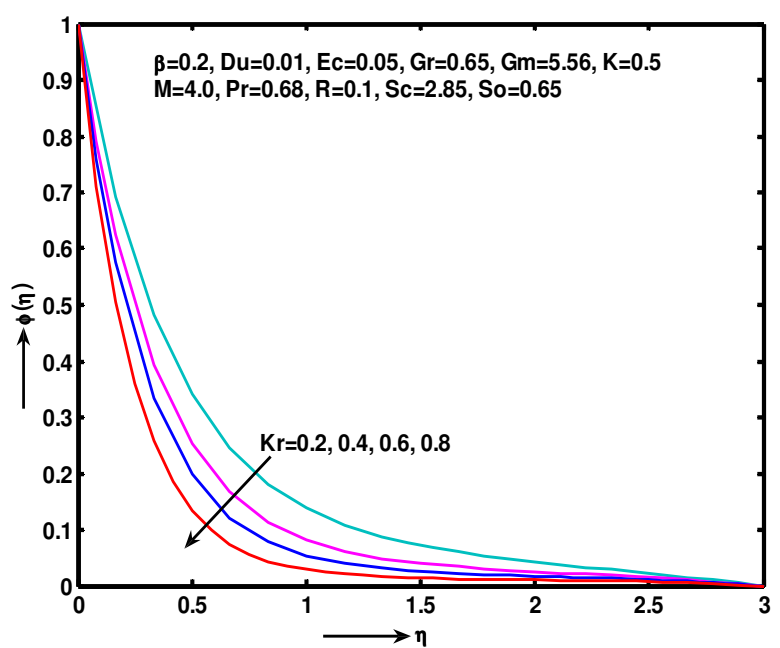

(c)

Fig. 3 Effects of $K r$ on (a) Velocity (b) Temperature (c) Concentration profiles
Figure 4 show the velocity profile for different values of magnetic field parameter $M$. It is observed that the velocity field decreases with increase of magnetic field parameter $M$ along the surface. These effects are much stronger near the surface of the plate. This indicates that the fluid velocity is reduced by increasing the magnetic field and confines the fact that the application of a magnetic field to an electrically conducting fluid produces a dragline force which causes reduction in the fluid velocity.

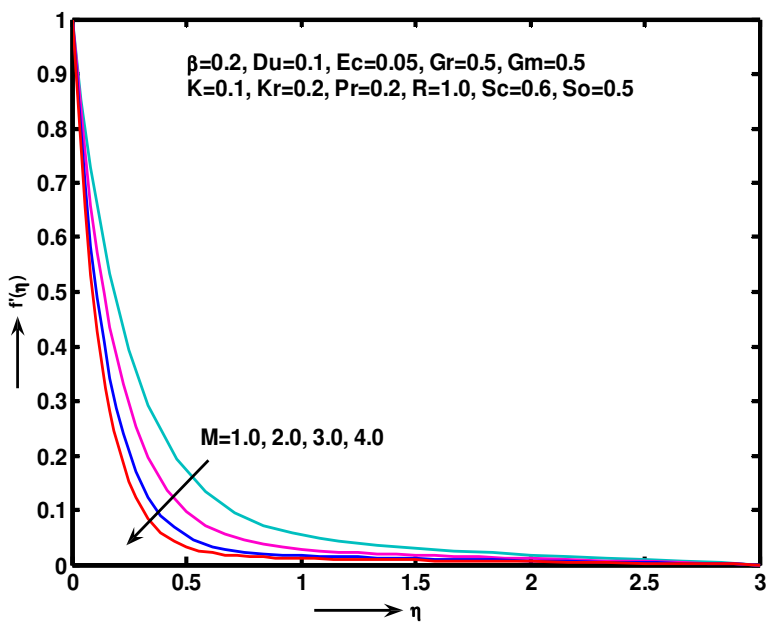

Fig. 4 Velocity profiles for various values of $M$

Figures 5(a)-5(c) are graphical representation of the velocity, temperature and concentration profiles for different values of the thermal radiation parameter $R$. It is revealed from these figures that both the velocity and concentration profiles decrease with increase in radiation parameter $R$ and then whereas reverse trend is observed in the case of temperature distribution. Physically speaking, the increase in radiation parameter means the release of heat energy from the flow region and boundary layer thickness becomes thinner.

Figures 6(a), 6(b), and 6(c), respectively, show the velocity, temperature and concentration field for different values of Soret number $S o$. It is observed from the figures that the velocity and concentration profiles increase with the increase of Soret number So, whereas reverse trend is observed in the case of temperature field. Physically, the fluid velocity rises due to larger thermal diffusion factor, since the higher Soret number stands for a larger temperature difference and precipitous gradient. Here $\beta=0$ gives the result for viscous incompressible fluid. From this it is observed that, velocity of the Newtonian fluid is greater than that of Maxwell fluid. Physically, high Deborah number flows correspond to solid-like behavior. These outcomes are same as noted in the Ref. (Sadeghy et al., 2005).

Figures 7(a)-7(c) illustrate the variations of skin friction coefficient, local Nusselt number and Sherwood number given by equations (17) and (18) with respect to magnetic field parameter $M$ and Dufour number $D u$. These figures show that these quantities increase almost linearly with $D u$. It is interesting to note that the effect of Dufour number on skin friction is little significant than the heat and mass transfer coefficients. This is due to the fact that an increase in $D u$ implies that strongly heat flows. Also we see that the skin friction increases with an increase of $M$ while the reverse trend is observed on the Nusselt number and the Sherwood number.

Figure 8 demonstrates the variation of the non-dimensional heat transfer coefficient with respect to So for different values of $D u$ and $E c$. From the graph we notice that the Nusselt number increases with an increasing $D u$ whereas the reverse trend is observed for $E c$. It is also observed that So has less effect on heat transfer coefficient for small values of $D u(0,0.2)$ and greater effect for larger values of $D u(0.4$, 
$0.5)$. From this we conclude that Eckert number $E c$ is the fluid motion controlling factor that supports the physical point of view.

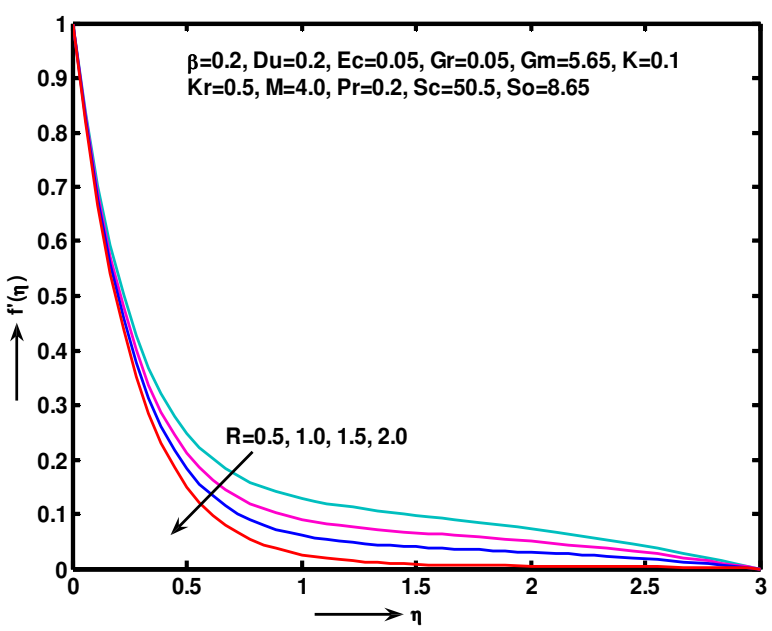

(a)

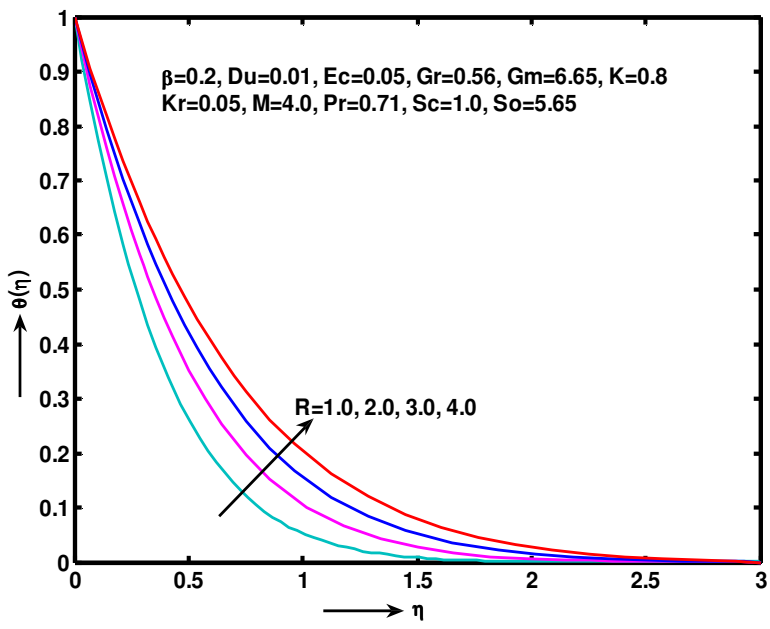

(b)

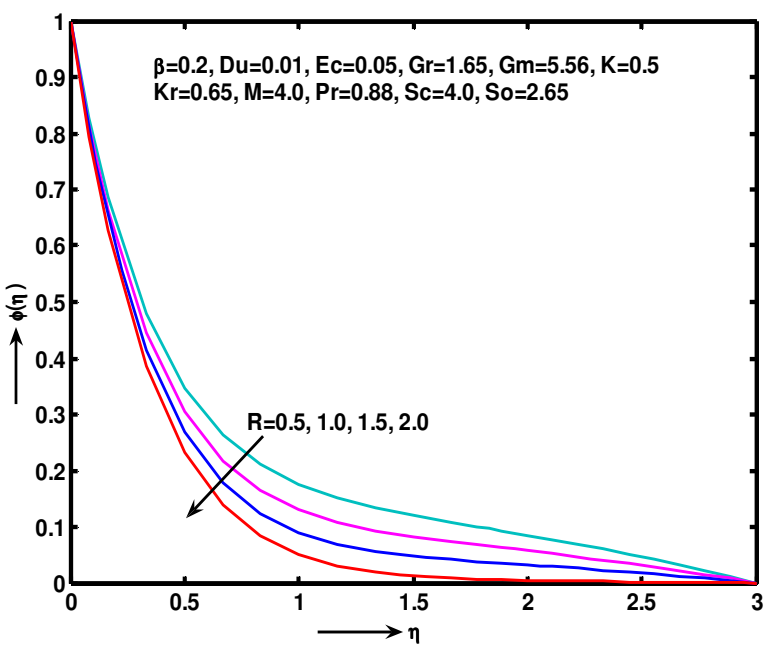

(c)

Fig. 5 Effects of $R$ on (a) Velocity (b) Temperature (c) Concentration profiles

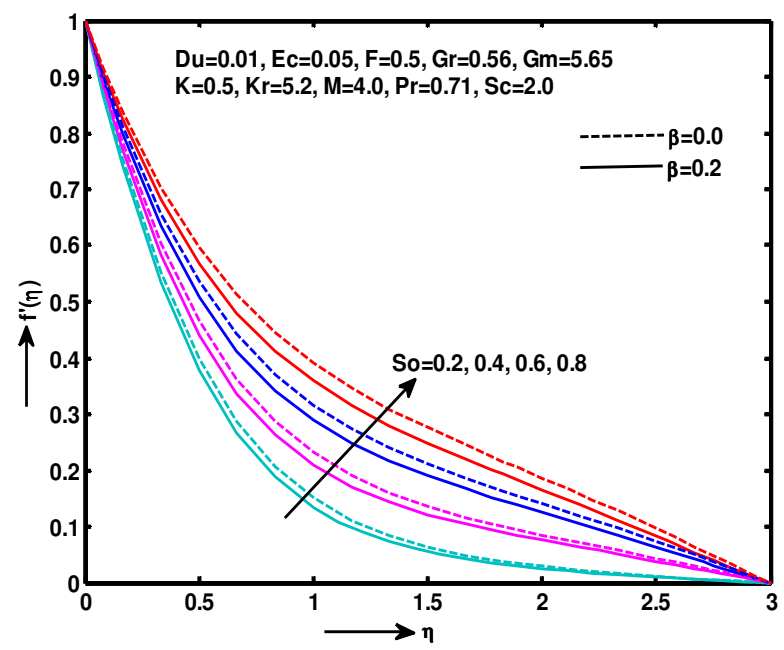

(a)

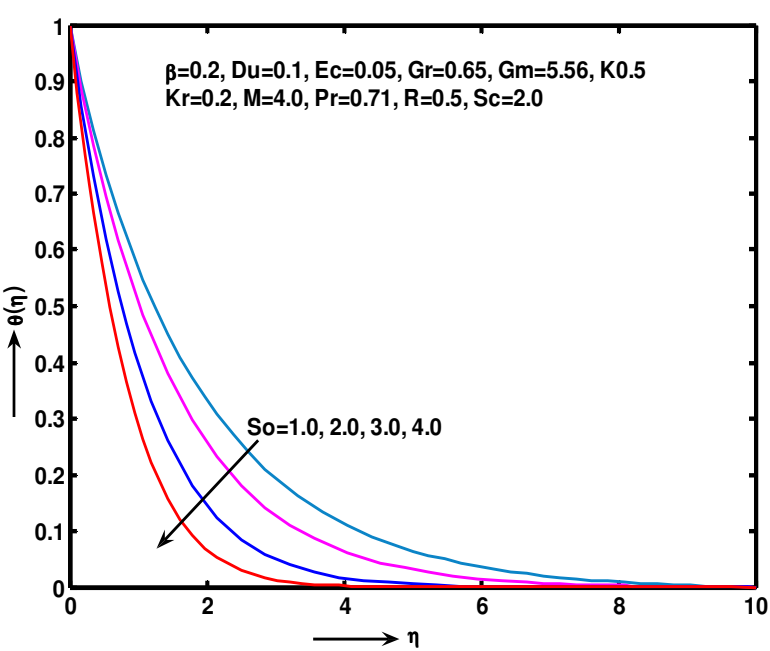

(b)

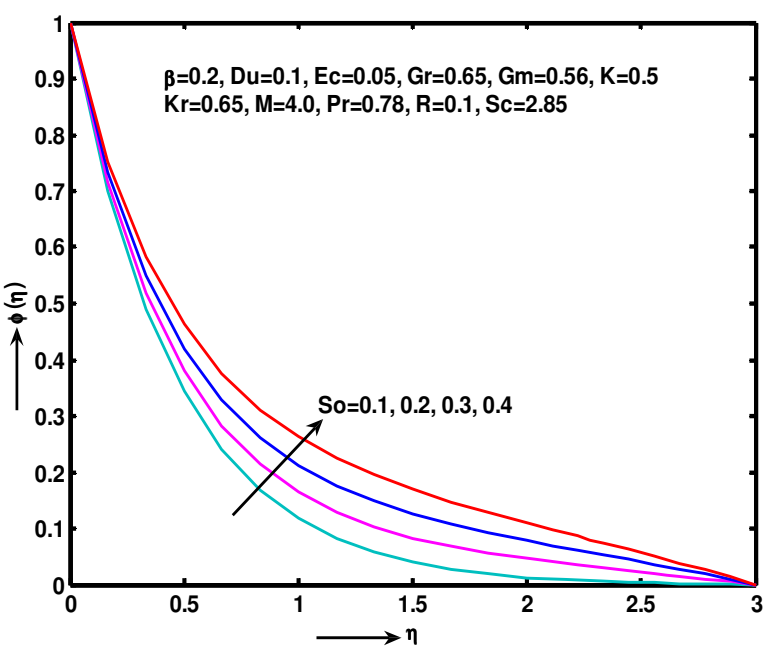

(c)

Fig. 6 Effects of So on (a) Velocity (b) Temperature (c) Concentration profiles 


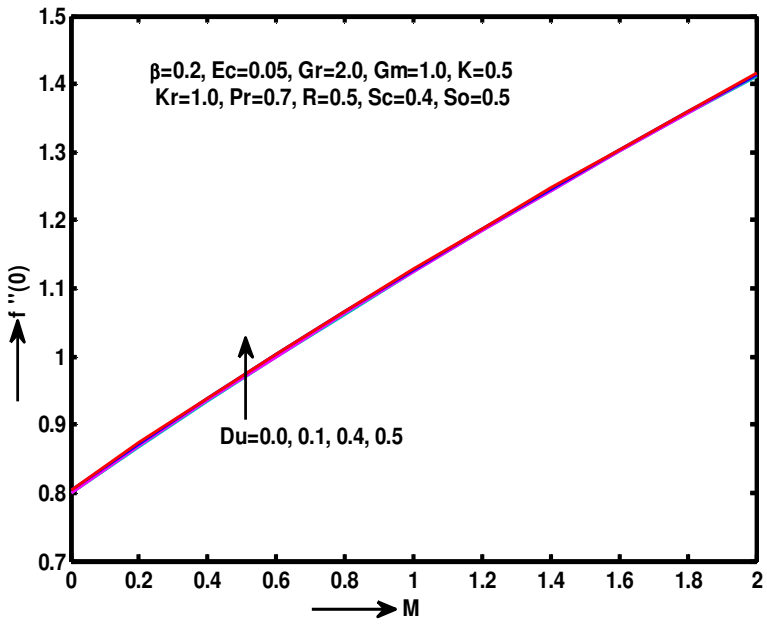

(a)

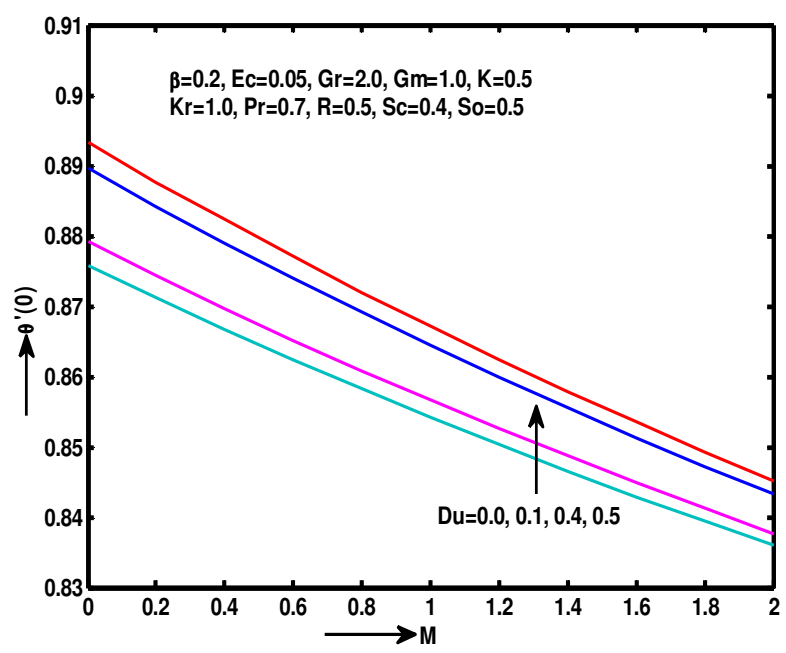

(b)

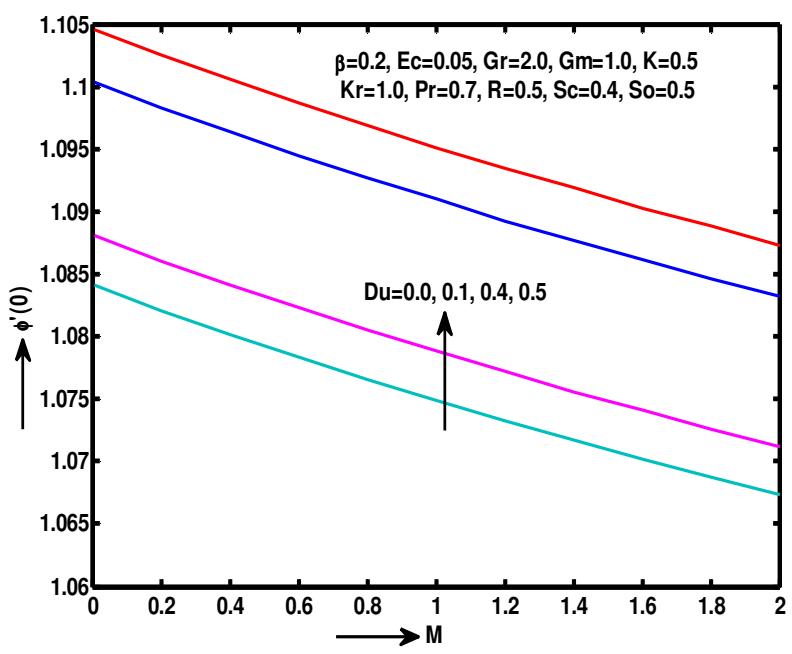

(c)

Fig. 7 Effects of $D u$ on (a) Skin friction coefficient (b) Nusselt number (c) Sherwood number

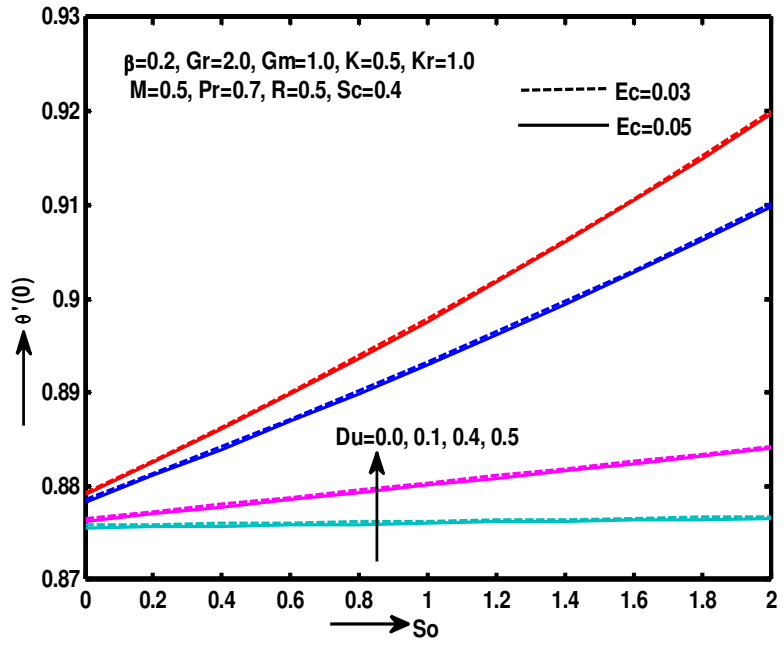

Fig. 8 Nusselt number for various values of $D u$

Table 2 shows the numerical values of the skin friction, Nusselt number and Sherwood number for different values of the Dufour number $D u$, Soret number $S o$ and Eckert number $E c$. It is seen that the skin friction coefficient, heat and mass transfer coefficients increase due to increase in $D u$ while decrease due to increase in $S o$ or $E c$.

Table 2: The values of skin-friction, Nusselt number Sherhwood for $\beta=0.2, G r=2.0, G m=1.0, K=0.5, K r=1.0, R=0.5, S c=0.4, M=1.0$

\begin{tabular}{|cccccc|}
\hline$D u$ & $E c$ & So & $f^{\prime \prime}(0)$ & $\theta^{\prime}(0)$ & $\phi^{\prime}(0)$ \\
\hline 0.0 & 0.02 & 1.0 & 0.9589 & 0.8724 & 0.9645 \\
0.1 & & & 0.9601 & 0.8764 & 0.9692 \\
0.4 & & & 0.9644 & 0.9212 & 0.9266 \\
& & & & & \\
& 0.1 & & 0.9641 & 0.9183 & 0.9287 \\
& 0.2 & & 0.9633 & 0.9097 & 0.9351 \\
& 0.3 & & 0.9617 & 0.9062 & 0.9402 \\
& & & & & \\
& & 0.5 & 1.1232 & 0.8677 & 1.0372 \\
& & 1.0 & 1.1231 & 0.8693 & 1.0253 \\
& & 1.5 & 1.1237 & 0.8719 & 1.0218 \\
\hline
\end{tabular}

Figures 9(a)-9(c) illustrate the variation of skin friction, Nusselt number and Sherwood number against thermal diffusion $S o$ for different values of $E c$, respectively. From these figures it is observed that, the skin friction and Nusselt number decrease with increasing of $E c$, while the reverse trend is observed on the Sherwood number. It is interesting to note that there is sharp decrease in the value of Sherwood number when thermal diffusion $S o$ is increased for all values of Eckert number.

\section{CONCLUSIONS}

In this paper, we study the Soret and Dufour effects on MHD flow of a Maxwell fluid past a vertical stretching sheet in the presence of chemical reaction. The governing partial differential equations are transformed into non-linear ordinary differential equations using a similarity transformation. The equations are solved numerically by using shooting method with Runge-Kutta fourth order scheme. The results are presented graphically and we conclude the following findings. 


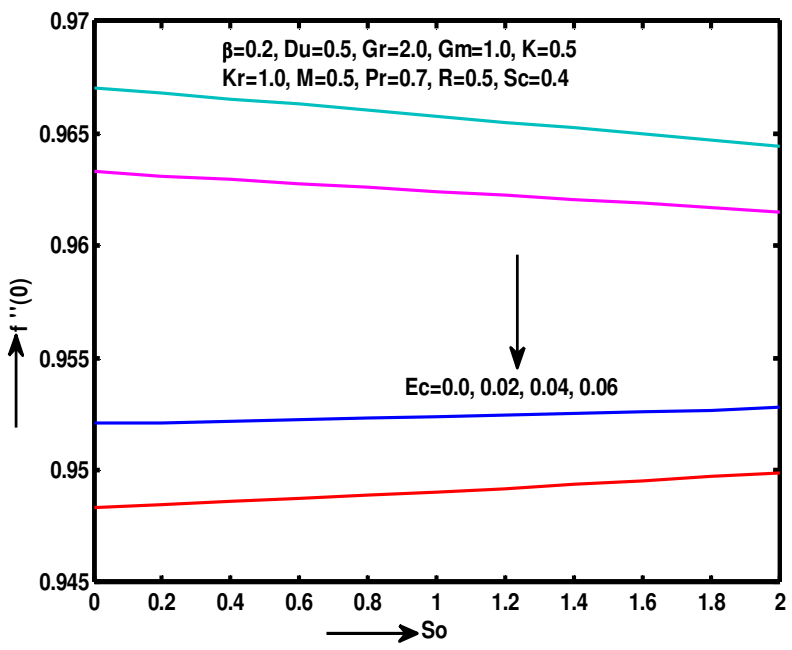

(a)

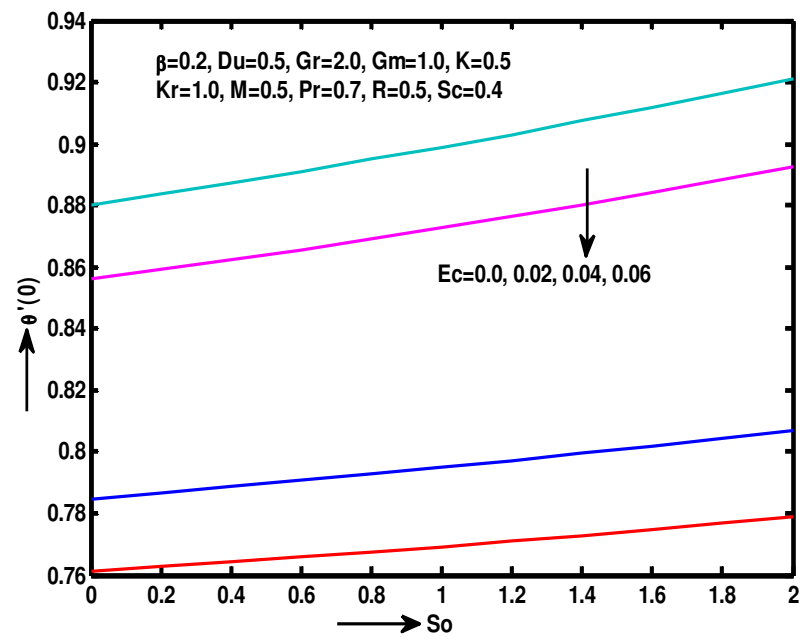

(b)

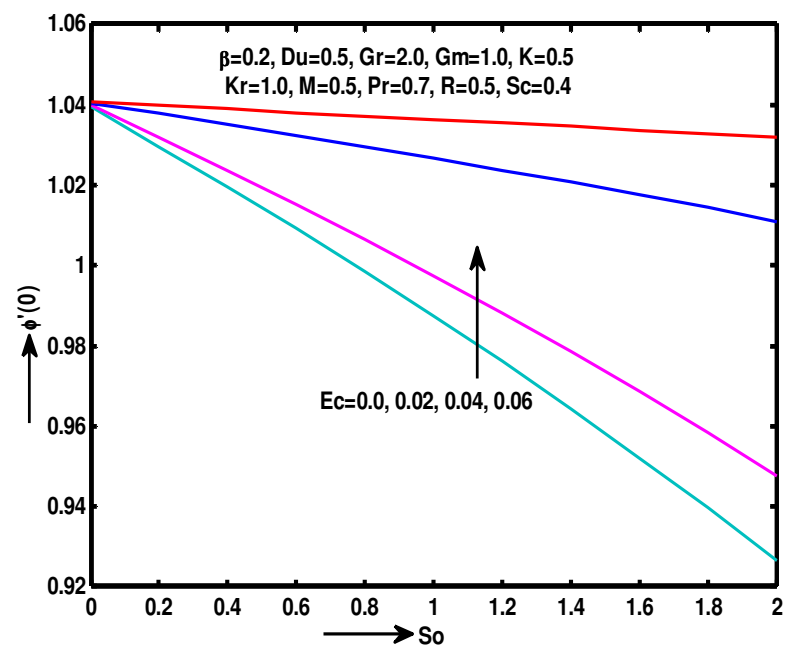

(c)

Fig. 9 Effects of $E c$ on (a) Skin friction coefficient (b) Nusselt number (c) Sherwood number (i) An increase in the Maxwell parameter $\beta$, radiation parameter $R$ and chemical reaction parameter $K r$ lead to decrease the velocity and concentration distributions but opposite effect occurs for the temperature profiles.

(ii) By increasing the Soret number So enhances the velocity and concentration profiles and decreases the temperature distributions.

(iii) The surface temperature and concentration of a sheet increase with increasing values of magnetic field parameter $M$, but the velocity decreases with an increase in magnetic field number $M$ due to the magnetic pull of the Lorentz force acting on the flow field.

(iv) A rise in Dufour number $D u$ enhances the skin friction coefficient, local Nusselt number and Sherwood number. This is due to strongly heat flow in the system.

(v) The effect of So on local Nusselt number and Sherwood number is opposite.

It is hoped that that the present investigation will provide a useful information for many scientific and industrial applications (such as polymer extrusion process) and also serve as a complement to the previous studies.

\section{ACKNOWLEDGEMENTS}

The authors express their intense gratefulness to the anonymous Reviewer for their valuable comments and suggestions.

\section{NOMENCLATURE}

a positive constant

$b \quad$ positive constant

$B_{0} \quad$ constant applied magnetic field

c positive constant

$C$ fluid concentration

$C_{f} \quad$ skin friction coefficient

$C_{p} \quad$ specific heat at constant pressure

$C_{s} \quad$ concentration susceptibility

$C_{w} \quad$ variable concentration

$C_{\infty} \quad$ uniform ambient concentration

$D \quad$ diffusion coefficient

$D_{m} \quad$ molecular diffusivity

Du Dufour number

Ec Eckert number

$g \quad$ acceleration due to gravity

$G_{r} \quad$ Grashof number

$G_{m} \quad$ modified Grashof number

$j_{w} \quad$ mass flux of the plate

$K$ permeability of the porous medium

$k$ thermal conductivity

$k^{*} \quad$ Rosseland mean absorption coefficient

$\mathrm{Kr} \quad$ chemical reaction parameter

$M \quad$ magnetic field parameter

$N u_{x} \quad$ local Nusselt number

Pr Prandtl number

$q_{r} \quad$ radiative heat flux

$q_{w} \quad$ heat flux from the plate

$R$ radiation parameter 
$\operatorname{Re}_{x} \quad$ local Reynolds number

Sc Schmidt number

So $\quad$ Soret number

Sh local Sherwood number

$T \quad$ fluid temperature (K)

$T^{4} \quad$ linear function of temperature

$T_{m} \quad$ mean temperature of the fluid

$T_{w} \quad$ variable temperature

$T_{\infty} \quad$ uniform ambient temperature

$(x, y)$ Cartesian coordinate's

$(u, v)$ velocity components along $\mathrm{x}$ and $\mathrm{y}$ axes

$U_{w} \quad$ velocity of stretching surface

Greek Symbols

$\beta$ Deborah number

$\beta_{C} \quad$ coefficient of expansion with concentration

$\beta_{T} \quad$ coefficient of thermal expansion

$v \quad$ kinematic viscosity

$\sigma$ electrical conductivity of the fluid

$\sigma^{*}$ Stefan-Boltzmann constant parameter

$\rho \quad$ fluid density

$\mu \quad$ dynamic viscosity

$\tau_{w} \quad$ skin friction

$\theta$ dimensionless temperature

$\phi \quad$ dimensionless concentration

\section{Subscripts}

$w \quad$ condition at the wall

$\infty \quad$ condition at free stream

\section{REFERENCES}

Abbas, Z., Wang, Y., Hayat, T., and Oberlack, M., 2010, "Mixed Convection in the Stagnation-point Flow of a Maxwell Fluid towards a Vertical Stretching Surface," Nonlinear Analysis: Real World Applications, 11(4), 3218-3228.

http://dx.doi.org/10.1016/j.nonrwa.2009.11.016

Abel, M.S., Tawade, J.V., and Nandeppanavar, M.M., 2012, "MHD Flow and Heat Transfer for the Upper-convected Maxwell Fluid over a Stretching Sheet," Meccanica, 47, 385-393.

http://dx.doi.org/10.1007/s1102-011-9448-7

Aliakbar, V., Alizadeh-Pahlavan, A., and Sadeghy, K., 2009, "The Influence of Thermal Radiation on MHD Flow of Maxwellian Fluids above Stretching Sheets," Com.Non-lin. Numer. Simul., 14, 779-794. http://dx.doi.org/10.1016/j.cnsns.2007.12.003

Alizadeh-Pahlavan, A., and Sadeghy, K., 2009, "On the Use of Homotopy Analysis Method for Solving Unsteady MHD Flow of Maxwellian Fluids above Impulsively Stretching Sheets," Common Non-linear Science and Numerical Simulat. 14, 1355-1365.

http://dx.doi.org/10.1016/j.cnsns.2008.03.001

Bilal Ashraf, M., Hayat, T., and Alhuthali, M.S., 2015, "ThreeDimensional Flow of Maxwell Fluid with Soret and Dufour Effects," Journal of Aerospace Engineering, 29(3).

http://dx.doi.org/10.1016/(ASCE)AS. 1943-5525.0000055
Bishnoi, J., and Goyal, N., 2012, "Soret Dufour Driven Thermosolutal Instability of Darcy Maxwell Fluid," IJE Transactions A: Basics, 25(4), 367-378

http://dx.doi.org/10.5829/idosi.ije.2012.25.04a.08

Chen, C.H., 1998, "Laminar Mixed Convection Adjacent to Vertical Continuously Stretching Sheets," Heat and Mass Tranf, 33, 471-76. http://dx.doi.org/10.1007/s002310050217

Fetecau, C., Athar, M., and Fetecau, C., 2009, "Unsteady Flow of Generalized Maxwell Fluid with Fractional Derivative due to a Constantly Accelerating Plate," Comput. Math. Appl., 57, 596-603. http://dx.doi.org/10.1016/j.camwa.2008.09.052

Fetecau, C., Jamil, M., Fetecau, C., and Siddique, I., 2009, "A Note on the Second Problem of Stokes for Maxwell Fluids," Int Journal of Non-Linear Mech., 44, 1085-1090.

http://dx.doi.org/10.1016/j.ijnonlinmec.2009.08.003

Hayat, T., and Qasim, M., 2010, "Influence of Thermal Radiation and Joule Heating on MHD Flow of a Maxwell Fluid in the Presence of Thermophoresis," International Journal of Heat and Mass Transfer, 53 ( 21), 4780-4788

http://dx.doi.org/10.1016/j.ijheatmasstransfer.2010.06.014

Hayat, T., Bilal Ashraf, B., Alsaedi, A., and Alhothuali, M.S., 2015, "Soret and Dufour Effects in Three-Dimensional Flow of Maxwell Fluid with Chemical Reaction and Convective Condition," Int Journal of Num Metds for Heat and Fluid Flow, 25 (1), 98-120. http://dx.doi.org/10.1108/HFF-11-2013-0322

Hayat, T., Abbas, Z., and Ali, N., 2008, "MHD Flow and Mass Transfer of a Upper-convected Maxwell Fluid Past a Porous Shrinking Sheet with Chemical Reaction Species," Physics Letters A, 372(26), 46984704.

http://dx.doi.org/10.1016/j.physleta.2008.05.006

Hayat, T., Abbas, Z. And Sajid, M., 2009, "MHD Stagnation-point Flow of an Upper-convected Maxwell Fluid over a Stretching Surface," Chaos, Solitons and Fractals, 39(2), 840-848.

http://dx.doi.org/10.1016/j.chaos.2007.01.067

Hayat, T., Awais, M., and Obaidat, S., 2012, "Mixed Convection, Three-Dimensional Flow of an Upper-Convected Maxwell Fluid under Magnetic Field, Thermal-Diffusion, and Diffusion-Thermo Effects," Journal of Heat Transfer, 134(4), 1-6.

http://dx.doi.org/10.1115/1.4005211

Hayat, T., Fetecau, C., and Sajid, M., 2008, "On MHD Transient Flow of a Maxwell Fluid in a Porous Medium and Rotating Frame," Phys. Lett. A 372, 1639-1644.

http://dx.doi.org/10.1016/j.physleta.2007.10.036

Hayat, T., Fetecau, C., Abbas, Z., and Ali, N., 2008, "Flow of a Viscoelastic Fluid with Fractional Maxwell Model Between Two Side Walls due to Suddenly Moved Plate," Non-linear Anal., Real World Appl., 9, 2288-2295.

Hayat, T., Sajjad, R., Abbas, Z., Sajid, M., and Hendi, A.A., 2011, "Radiation Effects on MHD Flow of Maxwell Fluid in a Channel with Porous Medium," Int Journal of Heat Mass Tranf, 54, 854-62. http://dx.doi.org/10.1016/j.ijheatmasstransfer.2010.09.069

Hayat, T., Shehzad, S.A., and Alsaedi, A., 2012, "Study on ThreeDimensional Flow of Maxwell Fluid over a Stretching Surface with Convective Boundary Conditions," Int Journal of Phy. Sci., 7(5), 761768 . 


\section{http://dx.doi.org/10.5897/IJPS11.1342}

Heyhat, M.M., and Khabazi, N., 2010, "Non-Isothermal Flow of Maxwell Fluids above Fixed Flat Plates under the Influence of a Transverse Magnetic Field," Proc. I Mech E Part C. J. Mech. Eng. Sci., 225.

http://dx.doi.org/10.1243/09544062JEMES2245

Motsa, S.S., Hayat, T., and Aldossary, O.M., 2012, "MHD Flow of Upper-Convected Maxwell Fluid over Porous Stretching Sheet using Successive Taylor Series Linearization Method" Applied Math. Mech.Engl. Ed., 33(8), 975-990.

http://dx.doi.org/10.1007/s10483-012-1599-x

Mukhopadhyay, S., and Bhattacharyya, K., 2012, "Unsteady Flow of a Maxwell Fluid over a Stretching Surface in Presence of Chemical Reaction," Journal of the Egyptian Mathematical Soc., 20, 229-234. http://dx.doi.org/10.1016/j.joems.2012.08.019

Mukhopadhyay, S., and Rama Subba Reddy, G., 2012, "Unsteady MHD Boundary Layer Flow of an Upper Convected Maxwell Fluid Past a Stretching Sheet with First Order Constructive/Destructive Chemical Reaction," Journal of Naval Arch. And Marine Eng., 9, 123133.

http://dx.doi.org/10.3329/jname.v9i2.12541

Mukhopadhyay, S., 2012, "Upper-Convected Maxwell Fluid Flow over an Unsteady Stretching Surface Embedded in Porous Medium Subjected to Suction/Blowing," Z. Naturforsch, 67, 641-646.

http://dx.doi.org/10.5560/ZNA.2012-0075

Nadeem, S., Rizwan U. Haq, and Khan, Z.H., 2014, "Numerical Study of MHD Boundary Layer Flow of a Maxwell Fluid Past a Stretching Sheet in the Presence of Nanoparticles," Journal of the Taiwan Institute of Chemical Engineers, 45(1), 121-126.

http://dx.doi.org/10.1016/j.jtice.2013.04.006

Noor, N.F.M., 2012, "Analysis for MHD Flow of a Maxwell Fluid Past a Vertical Stretching Sheet in the Presence of Thermophoresis and Chemical Reaction," World Acad. Sci., Eng. Technol., 64, 1019-1023.

Perez-Reyes, I., and Davalos-Orozco, L.A., 2011, "Effect of Thermal Conductivity and Thickness of the Walls in the Convection of a Viscoelastic Maxwell Fluid Layer," International Journal of Heat and Mass Transfer, 54(23), 5020-5029.

http://dx.doi.org/10.1016/j.ijheatmasstransfer.2011.07.016

Ramesh, C. and Rana, G.C., 2014, "Double Diffusive Convection in a Layer of Maxwell Viscoelastic Fluid in Porous Medium in the Presence of Soret and Dufour Effects," Journal of Fluids, 1-7.

http://dx.doi.org/10.1155/2014/479107

Ramesh, G.K., Gireesha, B.J., Hayat, T., and Alsaedi, A., 2016, "Stagnation Point Flow of Maxwell Fluid towards a Permeable Surface in the Presence of Nanoparticles," Alexandria Engineering Journal, 55(2), 857-865.

http://dx.doi.org/10.1016/j.aej.2016.02.007

Sadeghy, K., Najafi, A.H. and Saffaripour, M., 2005, "Sakiadis Flow of an Upper-Convected Maxwell Fluid', International Journal of NonLinear Mechanics, 40, 1220-1228.

http://dx.doi.org/10.1016/j.ijnonlinmec.2005.05.006
Nandy, S.K., 2015, "Unsteady Flow of Maxwell Fluid in the Presence of Nanoparticles toward a Permeable Shrinking Surface with Navier Slip," Journal of the Taiwan Institute of Chemical Engineers, 52, 2230.

http://dx.doi.org/10.1016/j.jtice.2015.01.025

Satya Narayana, P.V. and Venkateswarlu, B., 2016, "Heat and Mass Transfer on MHD Nanofluid Flow Past a Vertical Porous Plate in a Rotating System," Frontiers in Heat and Mass Transfer, 7, 8 http://dx.doi.org/10.5098/hmt.7.8

Satya Narayana, P.V., Venkateswarlu, B. And Venkataramana, S., 2015, "Thermal Radiation and Heat Source Effects on MHD Nanofluid Past a Vertical Plate in a Rotating System with Porous Medium," Heat Transfer-Asian Research, 44(1), 1-19.

http://dx.doi.org/10.1002/htj.21101

Satya Narayana, P.V., Venkateswarlu, B. And Venkataramana, S., 2013, "Effects of Hall Current and Radiation Absorption on MHD Micropolar Fluid in a Rotating system," Ain Shams Engineering Journal,, 4(4), 843-854.

http://dx.doi.org/10.1016/j.ase.2013.02.002

Satya Narayana, P.V., Venkateswarlu, B. and Venkataramana, S., 2013, "Chemical reaction and Radiation absorption effects on MHD micropolar fluid past a vertical porous plate in a rotating system," Journal of Energy Heat and Mass Transfer, 35, 197-214.

Shateyi, S., 2013, "A New Numerical Approach to MHD Flow of a Maxwell Fluid Past a Vertical Stretching Sheet in the Presence of Thermophoresis and Chemical Reaction," Boundary Value Prob, 196. http://dx.doi.org/10.1186/1687-2770-2013-196

Tan, W.C. and Masuoka, T., 2007, "Stability Analysis of a Maxwell Fluid in a Porous Medium Heated from below," Phys. Lett. A 360, 454-460.

http://dx.doi.org/10.1016/j.physleta.2006.08.054

Vajravelu, K., Prasad, K.V., Sujatha, A. and Chiu-on, N.G., 2012, "MHD Flow and Mass Transfer of Chemically Reactive Upper Convected Maxwell Fluid Past Porous Surface," Appl. Math. Mech.Engl. Ed., 33(7), 899-910.

http://dx.doi.org/10.1007/s10483-012-1593-8

Venkateswarlu, B and Satya Narayana, P.V., 2015, "Chemical reaction and radiation absorption effects on the flow and heat transfer of a nanofluid in a rotating system," Applied Nanoscience, 5, 351-360. http://dx.doi.org/10.1007/s13204-014-0324-3

Venkateswarlu, B. and Satya Narayana, P.V., 2016, "Influence of Variable Thermal Conductivity on MHD Casson Fluid Flow over a Stretching Sheet with Viscous Dissipation, Soret and Dufour Effects," Frontiers in Heat and Mass Transfer, 7, 16.

http://dx.doi.org/10.5098/hmt.7.16

Vieru, D., Fetecau, C. and Fetecau, C., 2008, "Flow of a Viscoelastic Fluid with Fractional Maxwell Model between two side Walls Perpendicular to a Plate," Appl Math. Comput., 200, 459-464.

http://dx.doi.org/10.1016/j.amc.2007.11.07 